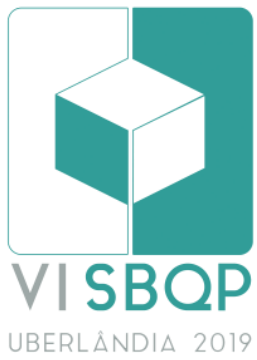

\title{
SUSTENTABILIDADE NOS APARTAMENTOS DO SÉCULO XXI
}

\author{
CALVET, Juliane \\ UnB, arq.julianecalvet@gmail.com \\ ABRAHÃO, Julia \\ UnB, julia.abrahao@gmail.com
}

\begin{abstract}
RESUMO
A personalização é a flexibilidade que envolve as necessidades dos clientes e o que as construtoras podem oferecer. No entanto, é uma estratégia que traz dificuldades para o sistema de produção, na medida em que o produto (unidade habitacional) deixa de ser padronizado. A personalização acaba por permitir modificações que não são definidas no projeto original, exigindo maior capacidade de resposta e maior integração entre os diferentes setores ou departamentos da empresa, além da necessidade de manter qualidade e prazos de entrega. Em virtude do crescimento das personalizações de apartamentos no Brasil, é necessário a busca de conhecimento sobre o assunto a fim de alcançar o desenvolvimento sustentável. A enorme geração de resíduos da construção civil, associada à deposição irregular, acarretam inúmeros impactos negativos para o meio ambiente, assim como no campo social e econômico. Neste contexto, o presente trabalho tem como objetivo, fazer um levantamento da demanda de apartamentos personalizados nos últimos cinco anos, em empresas que atuam nas cidades de Brasília-DF e Goiânia-GO. A metodologia contempla a revisão da bibliografia e posteriormente um questionário aplicado em seis empresas. Os resultados demonstram as demandas do projeto flexível e sua importância para o desenvolvimento sustentável.
\end{abstract}

Palavras-chave: Personalização de Apartamentos, Resíduos da Construção, Sustentabilidade.

\begin{abstract}
Personalization is the flexibility that comprises the client's needs and what building companies can offer. However, it is a strategy that causes difficulties to the production system, insofar as the product (housing unit) is no longer standardized. Personalization permits changes that are not defined in the original project, requiring greater responsiveness and integration between different sectors or departments of the company, as well as the need to maintain quality and deadline. Due to the growth of apartment customizations in Brazil, the search for knowledge on the subject is necessary in order to achieve a sustainable development. The enormous generation of construction waste, associated with irregular deposition, leads to numerous negative impacts on the environment, as well as the social and economic field. In this context, the present study aims to make a survey of the demand for personalized apartments in the last five years in companies operating in the cities of Brasilia-DF and Goiania-GO. The methodology includes a literature review and a questionnaire subsequently applied to six companies. The results demonstrate the demands of flexible design and its importance for sustainable development.
\end{abstract}

Key-words: Apartment Personalization, Construction Waste, Sustainability.

CALVET, J.; ABRAHÃO, J. Sustentabilidade nos Apartamentos do Século XXI. In: SIMPÓSIO BRASILEIRO DE QUALIDADE DO PROJETO NO AMBIENTE CONSTRUÍDO, 6., 2019, Uberlândia. Anais... Uberlândia: PPGAU/FAUeD/UFU, 2019. p. 231-238. DOI https://doi.org/10.14393/sbqp19023. 


\section{INTRODUÇÃO}

Na cadeia da indústria da construção, é necessário implantar progressivamente mecanismos visando a economia, satisfação do cliente e a qualidade. Segundo Strapasson (2011), a falta de flexibilidade nos projetos ou - não atendimento das necessidades dos usuários são causadores de intervenções, demolição parcial, ou mesmo demolição completa de uma edificação.

A indústria da construção é um dos setores da economia que mais geram resíduos, contribuindo para a degradação do meio ambiente e consumo de energia. A construção civil brasileira é responsável por gerar cerca de 122.262 toneladas de resíduos por dia, de acordo com o Panorama dos Resíduos Sólidos no Brasil publicado pela a ABRELPE - Associação Brasileira de Empresas de Limpeza Pública e Resíduos Especiais, em 2014.

Villa (2016), em publicação recente analisa o impacto ambiental, constatando que a indústria da construção é a atividade humana que gera maior impacto no meio ambiente - sobretudo no setor habitacional - e nos últimos anos, pesquisas visando reduzir este problema são cada vez mais frequentes.

Desde a década de 1990, observa-se mudanças na configuração dos empreendimentos visando atender um novo perfil de consumidor e novos estilos de morar. O setor da construção civil passou a se adequar às exigências desse novo mercado imobiliário, buscando responder às novas necessidades dos usuários, bem como à qualidade do produto a ser entregue.

Os construtores não podem mais ignorar a nova formatação da família contemporânea e suas necessidades. Um elemento essencial é a compreensão de que o estilo de vida -define seus gostos e exigências - é um atributo que varia como um processo de ciclo de vida dinâmico (FRIEDMAN; KRAWITZ, 1998). Sob a ótica sócio-cultural, o que enfatiza substancialmente o desenho e a conformação da habitação, são as transformações dos grupos sociais e soluções que contemplem suas necessidades materiais e simbólicas.

A flexibilização de projeto consiste na previsão e planejamento de alterações possíveis na planta (LARCHER, 2005). Enquanto que o conceito de personalização, deriva da possibilidade de atribuir ao produto ou serviço, características particulares do consumidor, visando atender sua satisfação Santana, Oliveira e Meira (2008). O processo de personalização se inicia a partir do momento em que o apartamento é vendido e o cliente solicita modificações tais como arranjo dos espaços e/ou materiais de acabamento e instalações Brandão e Heineck (2007).

Isto posto, e partindo do pressuposto que as personalizações nos apartamentos podem ter suas vantagens, desde que associada a um planejamento de custo, tempo e qualidade durante a obra. Buscou-se, nesta pesquisa, realizar um levantamento de dados que permitisse identificar, em duas cidades do Centro Oeste do Brasil, a demanda de personalização nos projetos de apartamentos em seis empresas incorporadoras nas cidades de Brasília-DF e Goiânia-GO.

\section{A INDÚSTRIA DE APARTAMENTOS}

Ao longo dos últimos anos a produção dos edifícios de apartamentos tem se concentrado nas mãos de empreendedores imobiliários. A lógica que permeia 
os projetos dos edifícios, nem sempre prioriza a qualidade destas habitações, primando em geral pelo lucro. Segundo o Instituto Brasileiro de Geografia e Estatística (IBGE-2010), o Brasil possui cerca de 6,1 milhões de apartamentos. 0 crescimento foi de $43 \%$ relacionado ao ano 2000, com um aumento significativo de empreendimentos lançados nos últimos anos nas cidades de médio e grande porte do país. A razão do aumento da verticalização deve-se a necessidade de atendimento das demandas habitacionais e a falta de espaços nos aglomerados urbanos.

Segundo Schwengber, 2015, "em decorrência do desenvolvimento urbano acelerado e das grandes obras da construção civil nas últimas décadas no Brasil, pode-se afirmar que os geradores dos resíduos englobam setores que vão desde a extração da matéria-prima e consequente produção dos materiais até a execução da construção em si por empresas da construção civil, denominadas de grandes geradoras de resíduos da construção".

Em segundo plano, encontra-se a geração de resíduos de pequenas obras realizadas por pessoas físicas e jurídicas em residências e pequenas empresas, sendo notório que as reformas oriundas da urbanização desordenada fazem com que as construções passem por adaptações e modificações aumentando a cadeia de resíduos.

Qualquer processo de produção gera resíduos, construir agredindo o meio ambiente e consumindo seus recursos naturais indiscriminadamente, ainda faz parte da sociedade industrial brasileira. Planejar e executar uma obra gerando um mínimo de perdas é essencial para que se tenha, um menor impacto ao meio ambiente.

Nesta visão os projetos passam a ser valorizados como a "espinha dorsal" do processo de produção. É cada vez menor o número de empresas construindo sem que haja um detalhamento correto e preciso do projeto do empreendimento, mesmo entre as de pequeno porte. Sendo assim, Adesse e Melhado (2003) afirmam - "conceber, projetar e construir um empreendimento implica na realização de inúmeras atividades técnicas, administrativas e financeiras, as quais precisam de uma efetiva coordenação para cada fase e entre elas, de modo a propiciar o resultado esperado em termos de prazo, qualidade e custos".

\section{A PERSONALIZAÇÃO NOS PROJETOS DE APARTAMENTOS}

A personalização é a possibilidade de flexibilidade que envolve as demandas dos clientes e o que as construtoras podem oferecer. No entanto, é uma estratégia que traz dificuldades para o sistema de produção, na medida em que $o$ produto (unidade habitacional) deixa de ser padronizado. A personalização acaba por permitir modificações que não estão definidas no projeto original, exigindo maior integração entre os diferentes setores ou departamentos da empresa, além da exigência de qualidade e prazos de entrega.

Segundo Santana, Oliveira e Meira (2008), pode-se dizer que personalizar é atribuir ao produto ou serviço, características subjetivas do consumidor. $O$ mesmo conceito se assemelha, ao conceito de customização, o qual se refere à habilidade de fornecer produtos e serviços diferenciados aos consumidores.

A demanda por residências personalizadas é encontrada de formas diversas. As diferenças se devem a fatores vários: necessidades individuais, sociais, demográficas, econômicas, novas configurações familiares, ciclo de vida, 
hábitos modernos (como o trabalho em casa, por exemplo). As modificações ocorrem em termos de espaço, área desejada, ambientes necessários, número de cômodos, disposição dos mesmos, equipamentos e instalações, materiais de acabamento e características estéticas. (FALCONI \& BRANDÃO, 2014)

$\mathrm{Na}$ indústria da construção brasileira, a personalização de unidades residenciais é uma realidade, e caso o projeto e a execução da obra não tenham sido planejadas estrategicamente para executar essa prática, pode acabar sendo mais um item gerador de resíduos sólidos. Um empreendimento personalizado é um produto mais complexo que o convencional, portanto requer maior atenção na administração de sua execução (CARVALHO, 2004). Nessa perspectiva, Falconi e Brandão (2014) apontam a "necessidade de traçar diretrizes para que por meio de uma administração adequada sejam evitados problemas como atrasos no cronograma e custos adicionais advindos de retrabalhos desnecessários".

As empresas têm descartado o velho paradigma da produção em massa por aplicação de tecnologias e novos métodos de gestão, criando variedades e personalização com flexibilidade e respostas rápidas.

Um dos grandes problemas gerados pela adoção da personalização relaciona-se às alterações de projetos solicitadas pelos clientes em fases mais adiantadas das obras, ocasionando impactos diretos nos custos e prazos, com alto grau de desperdícios de materiais e retrabalhos relacionados à mão de obra. A interação dos envolvidos no processo de personalização é, portanto, muito importante para a qualidade final do produto, mas muitas vezes isso não acontece no Brasil, ou acontece de modo falho. A deficiência no fluxo de comunicação entre os participantes acaba gerando as demais falhas de planejamento da construção.

As novas demandas que vêm surgindo na sociedade contemporânea, passam a exigir atendimentos cada vez mais individualizados, a flexibilidade não poderá mais ser encarada de forma reativa por parte das empresas de construção e deverá ter mais relevância a cada dia. Custo e qualidade consolidaram-se como objetivos de desempenho mais sólidos na construção, mas a flexibilidade também assume nos dias atuais um papel de importância no sistema de produção.

\section{MÉTODO DO ESTUDO}

A crescente demanda por variedade e a maior flexibilidade incentivam as empresas de construção de habitações a considerar novos tipos de projeto de produtos e processos, criando um estreitamento das relações entre cliente e construtora que passou a incluir solicitações para modificações do projeto, ou seja, fez-se emergir um novo condicionante no processo produtivo da indústria da construção civil: a personalização e a possibilidade de escolha (BRANDÃO, 2002).

Pela demanda, um fator preponderante de diversificação do morar envolve a evolução demográfica. Dados do mercado indicam que a distribuição de renda está cada vez mais dispersa, criando grandes diferenciais para disponibilidade de capital correspondente às necessidades e desejos dos consumidores, e a diversificação das configurações familiares nas décadas mais recentes variam conforme as diferenças de estágios do ciclo de vida familiar, os gostos pessoais, a cultura e os costumes regionais. 
Fatores como esses exigem um novo conceito no projetar e fazem com que o projeto original, aquele lançado e comercializado ainda 'na planta', difira em grande medida, surgindo, assim, a flexibilidade arquitetônica que, em outras palavras, é a flexibilidade do produto.

Para o entendimento do processo atual de personalização das empresas, se existe ou não a possibilidade de alterações nos apartamentos ofertados no mercado e a demanda dessas mudanças, aplicou-se um questionário em (06) seis construtoras/incorporadoras atuantes nos últimos cinco anos nas cidades de Brasília-DF e Goiânia-GO. Cada construtora lança em média quatro edifícios habitacionais por ano, considerando uma estimativa de quinze edifícios de classe média por construtora para a pesquisa.

O questionário aborda temas sobre a personalização de apartamentos considerando os seguintes aspectos: de que forma é divulgado e controlado o serviço de personalização, qual o momento em que as alterações podem ser solicitadas pelos usuários, o percentual de usuários que solicitam o serviço e quais as necessidades e preferencias de mudanças no projeto de personalização solicitadas.

\section{RESULTADOS E DISCUSSÃO}

Os dados coletados (tabela 01), apontam que atualmente é cada vez mais comum a personalização em apartamentos. As empresas não divulgam o serviço de personalização no material de divulgação, porém ao adquirir o apartamento $\mathrm{O}$ cliente pode solicitar a mudança na planta original, apresentando o projeto de modificação desenvolvido e assinado por um profissional da área. Ao solicitar as alterações, as empresas analisam a viabilidade e custo (cobrado do cliente) para executar o projeto de personalização, sendo um ponto positivo, pois tal procedimento minimiza o desperdício de material.

Em Brasília a solicitação de personalização do apartamento pode ser feita antes e durante a obra, já em Goiânia, das três construtoras contatadas, apenas duas permitem as modificações antes da obra, ou seja, após o início da obra não é mais permitido. Acredita-se que esse rigoroso prazo é devido ao planejamento dos gastos, compras de materiais e também aprovação dos projetos.

No caso de Brasília, uma empresa participante mencionou sobre a dificuldade de aprovação dos projetos personalizados e também da dificuldade na entrega do empreendimento. Esta conjuntura possivelmente explica a diferença das demandas entre as duas cidades. A demanda por personalização dos apartamentos em Goiânia é superior a 50\% e em Braślia inferior a 25\%. As variáveis são capazes de responder hipoteticamente a diferença das populações, mesmo sendo duas capitais tão próximas.

Estes dados sugerem a necessidade de aprofundar a pesquisa no sentido de compreender além das dificuldades legais do processo de personalização como também buscar respostas que permitam identificar de forma clara os elementos de projetos que não atendem as expectativas dos usuários. 
Tabela 1 - Dados fornecidos pelas empresas por meio do questionário aplicado.

\begin{tabular}{|c|c|c|c|c|c|}
\hline \multicolumn{3}{|c|}{ BRASÍLIA- DF } & \multicolumn{3}{|c|}{ GOIÂNIA-GO } \\
\hline EMPRESA 01 & EMPRESA 02 & EMPRESA 03 & EMPRESA 01 & EMPRESA 02 & EMPRESA 03 \\
\hline \multicolumn{6}{|c|}{ O MATERIAL DE DIVULGAÇÃO DOS EMPREENDIMENTOS APRESENTA COMO OPÇÃO DA EMPRESA IMÓVEIS PERSONALIZADOS? } \\
\hline SIM & NÃO & NÃO & NÃO & NÃO & NÃO \\
\hline \multicolumn{6}{|c|}{ EXISTE UM CONTROLE DAS ALTERAÇÕES DE PROJETO DOS IMÓVEIS PERSONALIZADOS? } \\
\hline SIM & SIM & SIM & SIM & SIM & SIM \\
\hline \multicolumn{6}{|c|}{ EXISTE UM PRAZO DAS ALTERAÇÕES DE PROJETO DOS IMÓVEIS PERSONALIZADOS? } \\
\hline SIM & SIM & SIM & SIM & SIM & SIM \\
\hline \multicolumn{6}{|c|}{ SE A RESPOSTA ANTERIOR FOR POSITIVA, EM QUAL MOMENTO OS PRAZOS SĀO EXIGIDOS PARA AS ALTERAÇÕES DE PROJETO? } \\
\hline DURANTE A OBRA & DURANTE A OBRA & DURANTE A OBRA & ANTES DA OBRA & ANTES DA OBRA & DURANTE A OBRA \\
\hline \multicolumn{6}{|c|}{ QUAL A PORCENTAGEM DE APARTAMENTOS QUE FAZEM PERSONALIZAÇĀO NOS EMPREENDIMENTOS? } \\
\hline $25 \%$ & $10 \%$ & $25 \%$ & $50 \%$ & $75 \%$ & $50 \%$ \\
\hline \multicolumn{6}{|c|}{ QUAIS AS PRINCIPAIS NECESSIDADES E PREFERÊNCIAS DO PRODUTO PERSONALIZADO? } \\
\hline $\begin{array}{c}\text { ALVENARIA } \\
\text { PONTOS ELÉTRICOS } \\
\text { MATERIAIS DE } \\
\text { ACABAMENTO }\end{array}$ & $\begin{array}{l}\text { ALVENARIA } \\
\text { MATERIAIS DE } \\
\text { ACABAMENTO }\end{array}$ & $\begin{array}{c}\text { ALVENARIA } \\
\text { PONTOS } \\
\text { ELÉTRICOS } \\
\text { MATERIAIS DE } \\
\text { ACABAMENTO }\end{array}$ & ALVENARIA & $\begin{array}{c}\text { ALVENARIA } \\
\text { PONTOS } \\
\text { ELÉTRICOS } \\
\text { MATERIAIS DE } \\
\text { ACABAMENTO }\end{array}$ & $\begin{array}{c}\text { ALVENARIA } \\
\text { PONTOS } \\
\text { ELÉTRICOS } \\
\text { MATERIAIS DE } \\
\text { ACABAMENTO }\end{array}$ \\
\hline
\end{tabular}

Fonte: autora (2018).

\section{CONCLUSÕES}

A geração de resíduos sólidos, assim como a quantidade de perdas, são problemas que tem sido repetido por décadas, como inerentes à Construção Civil. A bibliografia é farta sobre o assunto e aponta vários tipos de perdas e de erros de execução. O compromisso, com o meio ambiente e com a questão social, deve fazer parte do trabalho do arquiteto, do engenheiro e do construtor. Por isso as medidas de redução de qualquer item gerador de danos, se for computada em larga escala já fará diferença. Pensar em sustentabilidade é pensar no futuro, pois é para lá que estão sendo lançados os problemas criados hoje.

Os dados coletados demonstraram nas seis empresas a procura pela personalização de apartamentos, exigindo que as alterações sejam solicitadas durante a obra, significando que há demanda para o serviço e um prazo para que isso aconteça. Dessa forma, conclui-se que de alguma forma as modificações feitas contribuem para evitar uma possível reforma após a entrega do empreendimento e consequentemente reduz o desperdício de materiais, contribuindo com o meio ambiente.

As demandas relacionadas ao projeto de personalização continuarão a exigir estudos no que se refere aos produtos. O setor de construção já considera hoje a flexibilidade de produto como componente de sua estratégia de produção e competição. Com o aumento da demanda, passa a exigir atendimentos cada vez mais individualizados e a personalização não poderá mais ser encarada de forma reativa por parte das empresas de construção, deverá ter mais relevância a cada dia. Custo e qualidade consolidaram-se como objetivos de desempenho mais sólidos na construção, mas a personalização também deverá assumir um papel de importância no sistema de produção. Segundo os dados coletados foi possível averiguar a existência de um planejamento por parte das construtoras na oferta da personalização. É um serviço frequentemente solicitado pelos usuários, apontando uma demanda cada vez maior e evitando alterações posteriores a obra, contribuindo para a redução de resíduos da construção. 


\section{REFERÊNCIAS}

ADESSE, E.; MELHADO , S. B. A coordenação de projetos externa em empresas construtoras e incorporadoras de pequeno e médio portes. In: WORKSHOP BRASILEIRO DE GESTÃO DO PROCESSO DE PROJETO NA CONSTRUÇÃO DE EDIFÍCIOS, 3., 2003, Belo Horizonte. Anais... Belo Horizonte: UFMG, 2003.

ASSOCIAÇÃO BRASILEIRA DE EMPRESAS DE LIMPEZA PÚBLICA E RESÍDUOS SÓLIDOS. Panorama de resíduos sólidos no Brasil 2016. Disponível em http://www.mpdft.mp.br/portal/pdf/comunicacao/junho_2018/panoramaane xos2016.pdf

BRASIL. INSTITUTO BRASILEIRO DE GEOGRAFIA E ESTATISTICA. Censo Demográfico 2010. 2012. Disponível em <http://censo2010.ibge.gov.br/> Acesso em 13 dez 2012.

BRANDÃO, Douglas Queiroz. Personalização do produto habitacional: novas demandas e respostas das tecnologias de construção. ENCONTRO NACIONAL DE ENGENHARIA DE PRODUÇÃO, v. 22, p. 1-8, 2002.

BRANDÃO, D. Q.; HEINECK, L. F. M. Estratégias de flexibilização de projetos residenciais iniciadas na década de 1990 no Brasil: tão somente um recurso mercadológico? Ambiente Construído, Porto Alegre, v. 7, n. 4, p. 71-87, out./dez. 2007.

CARVALHO, L. O. Análise Qualitativa dos custos decorrentes da personalização de unidades habitacionais. 2004. 171 f. Dissertação (Mestrado em Engenharia Civil) - Universidade Federal de Santa Catarina, Florianópolis, 2004.

FALCONI, Taíssa Modesto Azevedo; BRANDÃO, Douglas Queiroz. Análise do processo de personalização de projetos de apartamentos na etapa de construção: estudo de caso. Gestão \& Tecnologia de Projetos, v. 9, n. 2, p. 71 95, 2014.

FRIEDMAN, Avi; KRAWITZ, David. The next home: Affordability through flexibility and choice. Housing and Society, v. 25, n. 1-2, p. 103-1 16, 1998.

FRUTOS, J. D. Desenvolvimento de interação ágil entre clientes empresas incorporadoras e construtoras de condomínios residenciais. 2000. 152 f. Dissertação (Mestrado em Engenharia) Universidade Federal do Rio Grande do Sul, Porto Alegre, 2000.

LARCHER, J. V. M. Diretrizes visando à melhoria de projetos e soluções construtivas na expansão de habitações de interesse social. 2005. $119 \mathrm{f}$. Dissertação (Mestrado em Engenharia Civil) - Universidade Federal do Paraná, Curitiba, PR, 2005.

SANTANA, C. L.; OLIVEIRA, D. N.; MEIRA, A. R. Caracterização de sistemas de personalização em construtoras da cidade de João Pessoa. In: ENCONTRO NACIONAL DE TECNOLOGIA DO AMBIENTE CONSTRUÍDO, 12., 2008, Fortaleza. Anais... ANTAC, 2008.

SCHWENGBER, Estela Regina. Resíduos da construção civil. Curso de especialização em Direito Internacional, Ambiental e Consumidor, Universidade Federal do Rio Grande do Sul, 2015.

STRAPASSON, D. C. Flexibilidade em projetos de edificações de ensino superior: estudo de caso na UFPR. 201 1. Dissertação (Mestrado em Engenharia Civil) Programa de Pós-Graduação em Construção Civil, Universidade Federal do Paraná, Curitiba, PR, 2011. 
VILLA, Simone Barbosa; ORNSTEIN, Sheila Walbe. Qualidade ambiental na habitação-avaliação pós-ocupação. Oficina de Textos, 2016. 